\title{
Effects of platelet rich plasma on ureteroneocystostomy in rabbits
}

\author{
Aibolat Smagulov, Nadiar Mussin
}

Department of Transplantation, West Kazakhstan Medical University, Aktobe Medical Center, Aktobe, Kazakhstan

Background: The aim of this study is to study the role of platelet-rich plasma (PRP) on the healing of ureteroneocystostomy (UCA) in a rabbit model.

Methods: A total of 14 adult male New Zealand rabbits (Oryctolagus cuniculus) were divided into two groups: group I $(n=7)$, standard anastomosis without PRP; group II $(n=7)$, standard anastomosis with PRP. The animals were observed for 7 days, then killed. The parameters of the study were complications of anastomosis, evaluation of hydroxyproline and histopathological evaluation of anastomoses.

Results: The level of hydroxyproline was statistically higher in the PRP group than in the control group $(P<0.05)$. Histological evaluation of the anastomoses showed almost complete healing in all animals. The average histological parameters of the animals in the groups did not differ.

Conclusions: According to our results, we assume that the use of PRP improves the healing of the anastomosis due to the level of hydroxyproline and a decrease in the inflammatory response. Further clinical studies are needed to confirm our hypothesis.

Corresponding author: Aibolat Smagulov

E-mail: smagulov.aibolat@gmail.com 